How different is this from Clerk Maxwell's top. Clerk Maxwell selected for his top the purest of paper and pigments. $\mathrm{He}$ endeavoured to match the spectral colours (considerably diluted). He selected a scarlet red with a tinge of orange like orange-red vermilion, lying in the spectrum one-third the way towards $D$, between the lines $C$ and $D$. His green was one-fourth the distance from $\mathrm{E}$, between $\mathrm{E}$ and $\mathrm{F}$, and resembled emerald green. $\mathrm{He}$ also selected a blue violet midway between $\mathrm{F}$ and $\mathrm{G}$, which was imitated by that purest of colours--ultramarine. Now let us try the given experiment under the favourable conditions guaranteed by Maxwell's discs, viz., the purest of colours painted on Whatman's paper. Taking up a disc of ultramarine and another of pale (not orange) chrome yellow, and concealing half of one disc behind the other, on rotating the compound disc so that the eye shall receive simultaneously blue and yellow light, the result is not white or even practically white, but a grey, tingrd with yellow. By a careful adjustment, hiding more of the yellow and exposing more of the blue (thereby altering the proportions of the text), it is possible to get rid of this yellowness and to obtain an absolutely neutral grey which it might be possible to persuade some grown-up people represented white, but which on analysis yields $7 I_{2}^{\frac{1}{2}}$ per cent. black to $28 \frac{1}{2}$ per cent. white. This may be proved by revolving a disc of black and white sectors in the above proportions, the results in each case being identical. But even this result, unsatisfactory as it is, does not apply to the passage quoted in the text, in which no special conditions are observed. I maintain what is easily proved by experiment in less time than it takes to write it, that when ordinary colours, e.g., gamboge and Prussian blue, are used, the residual light is green. ${ }^{1}$

I fear that already this letter is too long, and since I do not wish to monopolize the space kindly placed at the disposal of your correspondents, I must defer the consideration of the annotations on soap films. The other points are dealt with in the preface.

52 Lower Sloane Street, S. W., October 12 AMY JOHNSON.

I Do not think that the observations on my review of "Sunshine" require more than a very short answer.

I considered that the authoress had not by any means cleared the confusion which usually exists as to the meaning of the expression "mixing of colours." It is applied both to the case where two or more colours are seen superposed, e.s. by spinning coloured paper where the resultant tint is due to the sum of the separate colours in the constituents, and to the case of mixed pigments where the resultant tint is that which is common to the constituents. Now as the common "paint box" rule says that blue and yellow make green, that is that blue and yellow pigments mixed produce a green pigment, it seems to me very misleading to say "Cover half (of your top) blue and half yellow and you will see green." Of course it may happen that the slight departure from white which will be observed may be in a greenish direction, but it may also be inclined towards pink, or, for anything I know, towards any other colour. The one thing it will not do, however, is to make a green such as is obtained by mixing the pigments, and such as I fancy from the context any one would expect. C. V. B.

\section{The Photography of an Image by Reflection.}

THE great utility of spark photography for obtaining time records of quickly-moving objects must be apparent to all who know the experiments of Mr. C. Bell, Prof. Boys, and Lord Rayleigh. By means of spark photography the shadow of any object such as a jet of water, a flying bullet, or a broken soap film can be produced with perfect definition. The shadow of the moving object illuminated by an electric spark is thrown on to a sensitive plate in a dark room, and the plate is developed in the usual manner. The process of spark shadow photography will be found, I believe, of great service in physiological research. With a view to try this I attached a long sensitive plate to the traversing carriage of a chronograph ; the moving carriage closed and opened the primary circuit of an induction coil at pre-

I The purport of the experiment will be best understood if I state that it rollows a series of chapters on colour, viz.: the rainbow, the spectrum, its ecomposition by refraction and by reflection; while the last chapter discusses and explains, with experiments, the question of spectral lights versus habit of using, are then analysed by the prism, and found to be anything but monochromatic.

NO. I 20 I, VOL. 47$]$ arranged equal intervals of time. In front of the moving plate a frog's heart was placed in a slit on a screen; at each break a shadow of the heart was thrown on to the plate by means of the induced spark. By this means thirty positions of the heart were registered; the pictures were all sharp and clear. I have also used the same method for photographing the movements of insects.

Since these experiments; which I showed during the University Extension Meeting in Oxford this year, I have made several attempts to get spark photographs of the front view of objects (not their shadows). In my first experiments the objects were illuminated by an electric spark, the image being received on a plate in an ordinary camera. I found that so much useful light was shut off by the lenses that only a dim picture could be produced. A quartz lens was next tried and the results were rather better. I then determined to use nolens, but in its place a silvered mirror. A concave reflector made by silvering a concave lens of about Io c.m. diameter was so placed that it reflected the image of a white paper star $7 \mathrm{c} . \mathrm{m}$. diameter, revolving about 60 times in a second, on to an ordinary photographic plate, the total length traversed by the light being $80 \mathrm{c.m}$. The star was illuminated with a spark exactly similar to that used in the previous experiment; on development a good picture of the star came out. The reflector was neither well made nor well silvered. The idea was suggested by observing some spark photographs I obtained of waves on the surface of mercury reflecting light. When a steady light is used a photograph of any object is readily obtained by reflection from a suitable mirror. Probably a steel surface would be best. The mirror and plate were placed in a long box provided with a hole at one end through which the light reflected from the object passed. A few experiments made on living objects to test the time of exposure in Reflection Photography showed that in order to avoid over-exposure, a very rapid shutter must be used.

Trinity College, Oxford, October 25 . FREDERICK J. SMith.

\section{Induction and Deduction.}

As your correspondent invites discussion on this subject I hope you will allow me to repeat in a new form the views I expressed upon it in your columns some months ago. I quite agree with Mr. Russel in maintaining that "true induction is utterly unable to yield us any conclusion that is more than probable and approximate," understanding by induction inference from one or more special cases to a more general rule. But on the other hand it appears to me that Miss Jones's criticism is quite destructive of Mr. Russel's interpretation of geometrical teasoning. The point which both have missed I believe to be this, that a proposition stated in given words, such as the enunciation of Euclid's pons asinorum does not always and to every one convey the same information; and if it is meant in one sense its degree of reliability, and the method by which it must be proved, will be quite different from what they would be if it were meant in another. There are at least three different kinds of interpretation which may thus be put upon the proposition. It may mean (I) the triangle used to illustrate this proposition has equal sides; therefore it has equal angles; or (2) I have conceived a triangle which has equal sides, therefore I have conceived one which has equal angles; or (3) the connotation ascribed by the adjective "isosceles" implies the connotation "having equal sides."

It is not necessary for me here to dwell upon the distinction between the first two interpretations; but the difference between either of them and the third is that this latter gives us no information about any real thing or concept, but only about what is implied by using certain terms. And this latter kind of information clearly does not require to be based upon any real knowledge of things, but may be based solely on definitions of words. Arguments with propositions interpreted only in this sense are what I call symbolic arguments; and symbolic conclusions therefore give no real information unless they can be interpreted by the aid of real assertions, such as "I can conceive," or "There actually exist, things possessing the connotations ascribed to these terms by their definitions."

If this distinction has not before been recognized, it is because in most logical discussions we can in this way give a real meaning to our arguments. In elementary geometry, for example, we can-with more or less effort-conceive things, or even actually draw them, which answer to our definitions with sufficient accuracy. And, indeed, the reason why "Euclid" 